\title{
Percepción de consumidores frente a información nutricional de la carne bovina
}

\author{
Consumer perception of nutritional information in beef
}

\author{
Wilson Barragán ${ }^{1,2 *}$, Liliana Mahecha², Joaquín Angulo², Martha Olivera ${ }^{3}$
}

\begin{abstract}
RESUMEN
La carne bovina es considerada un alimento de alto valor biológico para el consumo humano, con aporte de proteína, grasa, vitaminas y minerales. La percepción del consumidor hacia la carne ha sido descrita como un fenómeno complejo, que se rige por las características intrínsecas y extrínsecas inherentes al producto. Con relación a la calidad nutricional, la percepción del consumidor es un factor muy importante, dado el creciente interés en conocer las propiedades nutricionales de los alimentos y la divulgación de la asociación del consumo de carnes rojas (grasa saturada) como factor de riesgo para enfermedades cardiovasculares, entre otras. Con el objeto de valorar la percepción del consumidor frente a la información nutricional de la carne bovina, se aplicaron y analizaron encuestas electrónicas a través de modelos de ecuaciones estructurales. Se obtuvo una tasa de recuperación del 18\% (108 encuestas), de las cuales $64.1 \%$ fueron hombres y 35.9\% mujeres. El mayor porcentaje de respuestas estuvo dado por personas con alto nivel académico (universitario con $43 \%$ y maestría con $39 \%$ ). Se identificaron seis variables latentes asociadas a la calidad de la carne, el consumo y preferencia de carne bovina, la importancia de nutrientes en alimentos, el estilo de consumo saludable, la aceptación de la carne bovina y la disposición a comprar carne con información nutricional. Entre los principales factores que determinan la calidad de la carne en la muestra encuestada se encuentran el color de la carne y el sitio de compra. La disposición a pagar por información nutricional de la carne estuvo influenciada principalmente por la necesidad de los consumidores del consumo de alimentos saludables.
\end{abstract}

Palabras clave: Calidad de carne, percepción, encuestas, modelos de ecuaciones estructurales

\section{ABSTRACT}

Beef is considered a food of high biological value for human consumption, with input of protein, fat, vitamins and minerals. The consumer's perception of meat has been described as a complex phenomenon, which is governed by the intrinsic and extrinsic characteristics inherent to the product. With regard to nutritional quality, consumer perception is a very important factor, given the growing interest in knowing the nutritional properties of foods and the dissemination of the association of the consumption of red meat (saturated fat) as a risk factor for diseases cardiovascular, among others. In order to assess the consumer's perception of beef nutritional information, electronic surveys were applied and analyzed through structural equation models. A recovery rate of $18 \%$ (108 surveys) was obtained, of which $64.1 \%$ were men and $35.9 \%$ were women. The highest percentage of responses were given by people with a high academic level (university with $43 \%$ and masters with $39 \%$ ). We identified six latent variables associated with: meat quality, beef consumption and preference, nutrient importance in foods, healthy eating style, acceptance of beef and the willingness to pay for meat with information nutritional. Among the main factors that determine the quality of the meat in the sample surveyed are the color of the meat and the place of purchase. The willingness to pay for nutritional information on meat was mainly influenced by the need of consumers for the consumption of healthy foods.

Key word: quality of meat, perception, surveys, models of structural equations

1 Corporación Colombiana de Investigación Agropecuaria - AGROSAVIA. Centro de Investigación Turipaná. Cereté.(Córd.), Colombia.

2 Universidad de Antioquia, Facultad de Ciencias Agrarias, Grupo de Investigación en Ciencias Animales - GRICA. Sede Robledo. Medellín - Antioquia, Colombia.

3 Universidad de Antioquia, Facultad de Ciencias Agrarias, Grupo de Investigación Biogénesis. Sede Robledo. Medellín Antioquia, Colombia.

* Autor por correspondencia: wbarraganh@agrosavia.co

Fecha de recepción: 29 octubre, 2017.

Fecha de aceptación: 06 septiembre, 2018.

DOI: 


\section{Introducción}

La carne es un alimento que ha estado ligado a la evolución de la humanidad, con un impacto significativo en el desarrollo cognitivo, morfofisiológico y en el entorno social (Pereira y Vicente 2017). Su importancia radica en la condición de ser un alimento catalogado como buena fuente de proteína, grasa, vitaminas (niacina, colina y B12), minerales (hierro, calcio, selenio y zinc) y de aminoácidos esenciales (fenilalanina, valina, treonina, triptófano, isoleucina, metionina, lisina y leucina) (Scollan et al., 2006; Pereira y Vicente 2017), con la particularidad de que aunque cada uno de estos componentes se puede encontrar por separado en varios alimentos, la carne es uno de los pocos que los proporciona de forma conjunta (Frank et al., 2017).

La percepción y la preferencia hacia el consumo de carne han sido definidas como un fenómeno complejo (Troy et al., 2010; Henchion et al., 2017), descrito como el resultado de un conjunto de comportamientos propios del consumidor, y de una serie de factores intrínsecos y extrínsecos inherentes al producto que actúan de manera sinérgica (Font-i-Furnols y Guerrero 2014). Diferentes autores han investigado sobre la percepción del consumidor de carne, y especialmente de carne bovina, frente a factores como la inocuidad del producto (Grunet et al., 2004) y el impacto ambiental (Li et al., 2016), e incluso sobre la percepción hacia la reducción del consumo de carne como una solución al cambio climático y su sustitución por fuentes de proteína alternas, tales como proteína cultivada e insectos (Cordts et al., 2014).

Con relación a la calidad nutricional, la percepción frente al consumo de carne bovina es un factor muy importante, dado el creciente interés del consumidor en conocer las propiedades nutricionales de los alimentos (Scollan et al., 2006) y la divulgación de la asociación del consumo de carnes rojas (grasa saturada) como factor de riesgo para enfermedades cardiovasculares, entre otras (Cordts et al., 2014).

En términos generales, la grasa como cualidad intrínseca y atributo nutricional de la carne se ha descrito como un factor negativo sobre la percepción de los consumidores (Grunet et al., 2004; Henchion et al., 2017). Sin embargo, este concepto ha sido sobrevalorado dado que existe evidencia poco divulgada que revela la importancia de la grasa proveniente de rumiantes, y específicamente de sus componentes, en la salud humana (Sales-Campos et al., 2013).
Según la USDA (2016), la carne bovina presenta una concentración de ácidos grasos monoinsaturados (MUFA) mucho mayor que la de ácidos grasos saturados (SFA), incluso en carne magra (14,5\% más MUFA) y en carne de animales con gran proporción de grasa como la raza Wagyu (40,4\% más MUFA), con una clara tendencia a predominio del ácido graso oleico (C18:1 n9). Este ácido graso monoinsaturado ha sido descrito como un potente agente regulador del sistema inmune y de la respuesta inflamatoria en enfermedades autoinmunes, regulador del colesterol plasmático, modulador de los comportamientos trombóticos de las plaquetas y como agente protector del cáncer de seno (Sales-Campos et al., 2013). En términos de ácidos grasos poliinsaturados (PUFAS), la carne bovina aporta un ácido graso exclusivo de rumiantes (rumenico C18:2 cis9-tras11), el cual también se ha descrito como un agente antiarteriosclerótico, antiinflamatorio, antidiabético y, sobre todo, anticarcinogénico, así como potenciador del sistema inmune (Frank et al., 2017).

Con relación a la percepción de calidad y experiencia de consumo, la grasa de la carne y específicamente la intramuscular, juega un rol importante en la mejora de la palatabilidad, contribuye con una mayor experiencia en la masticación, jugosidad, sabor y terneza (Henchion et al., 2017; Joo et al., 2013; Grunet et al., 2004).

Para el caso colombiano, la percepción sobre el consumo de carne bovina es aún incipiente, dejando de lado la importancia que tiene para la industria cárnica entender los factores que motivan al consumidor a tomar la decisión de compra de este producto (Troy et al., 2010), lo cual resulta prioritario, de cara a la actual era digital y a la facilidad de acceso a medios, que ha contribuido con la masificación de la información, en muchos casos negativa hacia el consumo de carne bovina (Frank et al., 2017).

En este contexto, el presente trabajo tuvo como objetivo valorar la percepción del consumidor frente a la información nutricional de la carne bovina, mediante la aplicación de encuestas electrónicas y la modelación del fenómeno a través de modelos de ecuaciones estructurales.

\section{Materiales y métodos}

Con base en las experiencias publicadas por Grunet et al. (2004) y Font-i-Furnol y Guerrero (2014), se diseñó una encuesta estructurada, con cuatro ejes: 1) aspectos socioeconómicos 2) preguntas 
relacionadas con la percepción de la calidad de la carne 3) costos y preferencias de cortes de carne y 4) disposición a pago por etiqueta de calidad de carne bovina. Las bases teóricas de los aspectos asociados a calidad de la carne fueron tomadas de las revisiones publicadas por Joo et al. (2013), Troy et al. (2010) y Henchion et al. (2017).

El cuestionario fue diseñado en la plataforma Google Forms ${ }^{\circledR}$, la cual ha sido descrita como un sistema informático gratuito que permite a los usuarios crear formularios para recopilación de datos de forma colaborativa. En el encabezado del formulario se diseñó una advertencia de consentimiento informado que señalaba que la información aportada era totalmente confidencial y que solo sería usada para fines académicos. En caso de que el receptor decidiera no aceptar la encuesta, el formulario se cerraba sin permitir ser aplicado.

El cuestionario contó con preguntas (5) de selección única para el componente socioeconómico y de disposición a pago por información de calidad. Tipo Likert (24) en escala 1-5, donde uno era que estaba en desacuerdo con la afirmación y cinco correspondía a totalmente de acuerdo con la afirmación, para las preguntas relacionadas con el componente de calidad de la carne; y pregunta abierta para preferencia de cortes y valor pagado por un $\mathrm{kg}$ de carne (2) en $\$ \mathrm{COP}$ (pesos colombianos).

La aplicación de la encuesta se realizó con base en un muestreo no probabilístico por conveniencia, dada la ausencia de universo muestral (ausencia en la cuantificación de consumidores de carne bovina en Colombia), su bajo costo (Navarrete 2000) y facilidad de uso de una lista cerrada de correos electrónicos. Este método de muestreo es común en la aplicación de investigaciones de comportamiento, y según lo descrito por Gravetter y Forzano (2015), una forma de ajustar los alcances del muestreo se da por la generación de variabilidad entre la muestra tomada. Para este trabajo la variabilidad estuvo dada por sexo (masculino, femenino), nivel educativo superior (desde nivel primaria hasta doctorado), ingresos económicos (desde un salario mínimo en Colombia hasta más de cinco salarios mínimos, SMMV), frecuencia de consumo de carne (desde nunca hasta mayor a cuatro veces por semana) y región natural de Colombia (Andina, Caribe, Pacífico, Amazonia y Orinoquía).

La recolección de la información se llevó a cabo entre el 3 de abril y el 5 de mayo de 2017. En total, se enviaron 602 correos con formularios para respuesta, de los cuales se recuperaron 108. La base de datos generada automáticamente por la plataforma Google Forms ${ }^{\circledR}$ fue almacenada en formato de valores separados por comas (CSV) para su posterior manejo en software estadístico, revisión y depuración. Para el caso de variables como disposición a pago (sí, no) y frecuencia de consumo de carne bovina, se utilizaron variables auxiliares para su análisis.

Para el análisis de la información se abordó la metodología de modelos de ecuaciones estructurales (MEE), también llamado análisis de estructuras de covarianza o análisis de variables latentes (Kline, 2011), el cual consiste en una metodología de análisis multivariado que combina aspectos de regresión múltiple (examina relaciones de dependencia) y de análisis factorial (representa factores o conceptos no medidos, a partir de múltiples variables), con el objeto de evaluar las relaciones de interdependencia o dependencia que se puedan generar en los fenómenos estudiados (Hair et al., 2010).

La base de datos para el análisis factorial exploratorio se consideró a partir de la existencia de correlaciones en las variables mayores a 0,3 como valor absoluto. Estas variables fueron sometidas al criterio de medida de adecuación muestral (MSA) de Kaiser-Meyer-Olkin (Hair et al., 2010), descartando las variables referentes al empaque de la carne y a la limitación por consumo de grasa, las cuales obtuvieron un valor de MSA <0,5. Posteriormente se empleó un análisis factorial exploratorio mediante la librería psych, con la utilización del método de máxima verosimilitud para extraer los factores y la rotación varimax. Este proceso permitió identificar cuatro constructos, los cuales fueron validados a través del Alfa de Cronbach (Hair et al., 2010) con un criterio de aceptación $>0,6$.

Posteriormente se aplicó un análisis de MEE a través de la librería lavaan, mediante el cual se especificaron las relaciones de interdependencia entre las variables latentes y las variables observadas, se generaron nuevas variables latentes (aceptación de la carne bovina, disposición a pago por información de calidad), y se ajustaron las varianzas y covarianzas en las variables. Los resultados se presentaron mediante un diagrama de senderos.

Para la validación de los resultados se consideró: ausencia de varianzas negativas en el error, correlaciones mayores a uno, grandes errores estándar y cargas factoriales $>1$. Para la validación de los estadísticos de bondad de ajuste en el MEE, se validaron índices de ajuste absoluto como el cuadrado 
medio estandarizado del residual (SRMR por sus siglas en inglés) y el cuadrado medio del error por aproximación (RMSEA por sus siglas en inglés), con un criterio de aceptación $<0,08$ y los índices de ajuste comparativo tales como CFI (índice de ajuste comparativo) y TLI (índice Tuker-Lewis), con un valor de aceptación >0,9 (Hair et al., 2009; Kline et al., 2011).

Para el desarrollo de los análisis estadísticos se empleó el software R en su versión 3.3.2 liberado bajo el nombre Sincere Pumpkin Patch (R Core Team 2016).

\section{Resultados}

Se alcanzó una tasa de respuesta del $18 \%$ (108/602). Esta muestra estuvo conformada en su gran mayoría por hombres $(64,1 \%)$ y en menor proporción por mujeres $(35,9 \%)$. El mayor porcentaje de respuestas se obtuvo de personas en las regiones Caribe y Andina (88\%). Así mismo, el mayor porcentaje de respuestas estuvo dado por personas con un nivel académico superior (universitario con $43 \%$ y maestría con 39\%) y un ingreso económico mensual mayor a 5 (38\%) SMMV (> 3,6 millones de pesos COP).

El análisis de MEE presentó unos índices de bondad de ajuste que validaron su interpretación. Para los índices de ajuste absoluto, el SRMR presentó un valor de 0,072 . En el caso de los índices de parsimonia, el RMSEA presentó un valor de 0,038 . Por último, los indicadores de bondad de ajuste comparativo CFI y TLI presentaron valores satisfactorios de 0,964 y 0,956 , respectivamente. Estos resultados concuerdan con la validación de los modelos de ecuaciones estructurales citadas por Hair et al. (2009) y Kline et al. (2012).

La Tabla 1 presenta los resultados obtenidos del análisis de MEE, en el cual se observan las cargas factoriales y los índices de fiabilidad para validación y convergencia del modelo. El diagrama de senderos se muestra en la Figura 1.

La revisión del color de la carne al momento de la compra y la preferencia por la compra de carne en el mismo sitio presentaron las mayores Cargas Factoriales (CF) con valores de 0,677 y 0,573, respectivamente, en la variable latente (VL) "calidad intrínseca de la carne". Contrario a esto la revisión del contenido de grasa tuvo poca contribución (CF 0,390).

LaVL "consumo y preferencia de la carne bovina" estuvo conformada por cinco variables observadas, de las cuales la preferencia por la carne de res sobre otras carnes, la mayor confianza en el consumo de carne de res que en carne de pollo o cerdo y la frecuencia de consumo en la carne de res presentaron las mayores CF. Con menor CF se ubicó la variable que indagó sobre la consideración de la carne de res como una buena fuente de nutrientes.

En el caso de la VL "importancia de nutrientes (grasa, proteína, perfil AG)", los resultados indicaron que los encuestados se preocupan por revisar la información nutricional de los alimentos que compran (CF 0,835$)$ y el contenido de nutrientes como grasa y proteína en los alimentos (CF 0,841). Sin embargo, aunque los encuestados ven como positivo revisar los contenidos nutricionales de los alimentos que consumen, este comportamiento no es relevante al momento de preferir la carne de res si esta declarara sus contenidos de grasa, proteína y perfil de ácidos grasos (CF 0,335).

Con relación a la VL "estilo de consumo saludable", los encuestados coincidieron en valorar el consumo de alimentos saludables y comprar alimentos saludables para sí mismos y para sus familiares como factores importantes en la construcción de este concepto.

En la VL “aceptación de la carne bovina” formada a partir de variables latentes y variables observadas, la VL "consumo y preferencia por la carne bovina" no presentó CF significativa ( $>0,05)$, como tampoco la variable observada que consideró que la carne de res es una buena fuente de nutrientes $(\mathrm{p}>0,05)$. Contrario a esto, se evidenció una CF significativa $(0,598)$, relacionada con la calidad intrínseca de la carne, lo cual indica que las características de color de la carne y confianza en el sitio de compra principalmente pueden determinar la aceptación del producto.

La última VL evaluada fue la "disposición a comprar carne bovina con información de calidad nutricional", la cual presentó mayor peso dado por las variables latentes "importancia de nutrientes en los alimentos" (CF 0,935) y "estilo de consumo saludable" (CF 0.601). Para esta VL no se presentó efecto significativo de la $\mathrm{CF}$ en "aceptación de la carne bovina". Este resultado sugiere que la motivación a pagar más dinero por información nutricional de la carne de res (68\% de los encuestados) estaría influenciada por la preocupación de los consumidores por comprar alimentos que se relacionen con características nutracéuticas, las cuales han conocido por experiencia propia o por experiencias adquiridas a través de otras personas, 


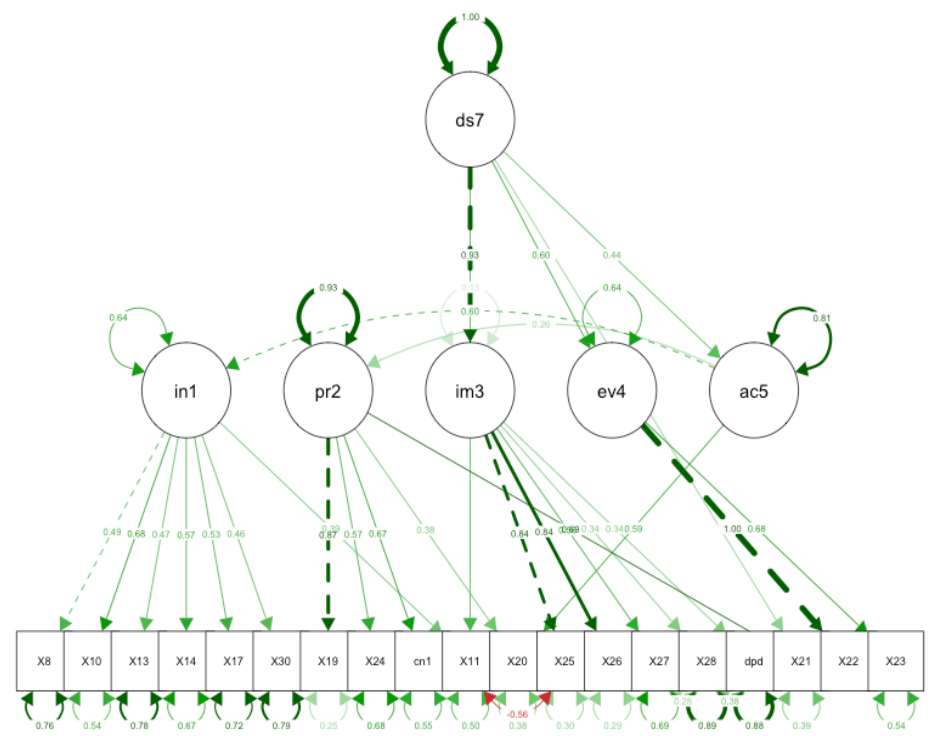

Figura 1. Modelo de ecuaciones estructurales para la percepción de consumidores frente información nutricional en la carne bovina.

Tabla 1. Variables latentes en la percepción de los consumidores frente información nutricional en la carne bovina.

\begin{tabular}{|c|c|c|c|c|}
\hline Latente & Variable independiente & $\begin{array}{c}\text { Carga } \\
\text { Factorial }\end{array}$ & Valor $\mathbf{p}$ & $\begin{array}{c}\text { Alfa de } \\
\text { Cronbach }\end{array}$ \\
\hline \multirow{5}{*}{$\begin{array}{l}\text { Consumo y preferencia por la } \\
\text { carne bovina (Tradición) }\end{array}$} & Prefiero carne de res sobre otras carnes & 0,868 & 0,001 & \multirow{5}{*}{0,796} \\
\hline & Considero carne de res buena fuente de nutrientes & 0,385 & 0,003 & \\
\hline & Me da más confianza comer carne de res, que de pollo o cerdo. & 0,690 & 0,001 & \\
\hline & Me da más confianza comer carne de res que de pescado. & 0,565 & 0,001 & \\
\hline & Frecuencia de consumo & 0,669 & 0,001 & \\
\hline \multirow{5}{*}{$\begin{array}{c}\text { Importancia de nutrientes en los } \\
\text { alimentos (grasa, proteína, perfil } \\
\text { AG) }\end{array}$} & Cuando compro un alimento, reviso su información nutricional & 0,835 & 0,001 & \multirow{5}{*}{0,753} \\
\hline & Me importa el contenido de grasa y de proteínas que tienen los alimentos. & 0,841 & 0,001 & \\
\hline & $\begin{array}{c}\text { Reviso el contenido de ácidos grasos omega } 3 \text { y } 6 \text {, colesterol y ácidos grasos } \\
\text { saturados en los alimentos }\end{array}$ & 0,561 & 0,001 & \\
\hline & $\begin{array}{l}\text { Si la carne de res declarara el contenido de ácidos grasos instaurado (omega } 3 \text { y } \\
\text { omega 6), me gustaría más }\end{array}$ & 0,335 & 0,001 & \\
\hline & Disposición a pago & 0,343 & 0,001 & \\
\hline \multirow{7}{*}{ Calidad intrínseca de la carne } & La suavidad de la carne es fundamental cuando voy a comprar & 0,493 & 0,001 & \multirow{7}{*}{0,713} \\
\hline & Reviso el color cuando compro la carne res & 0,677 & 0,001 & \\
\hline & Cuando compro carne me fijo en su contenido de grasa & 0,390 & 0,001 & \\
\hline & La facilidad de cocinar determina el tipo de carne que compro & 0,469 & 0,001 & \\
\hline & Prefiero comprar la carne en lugares reconocidos & 0,573 & 0,001 & \\
\hline & Usualmente compro la carne en el mismo sitio & 0,529 & 0,001 & \\
\hline & Cuando compro carne de res, escojo la que esté más magra & 0,46 & 0,001 & \\
\hline \multirow{2}{*}{ Estilo de consumo saludable } & Me preocupo por comer dietas saludables & 0,998 & 0,001 & \multirow{2}{*}{0,808} \\
\hline & Compro alimentos que sean los más saludables para mí y para mi familia & 0,676 & 0,001 & \\
\hline \multirow{3}{*}{ Aceptación de la carne bovina } & Calidad intrínseca de la carne & 0,598 & 0,001 & \multirow{3}{*}{-} \\
\hline & Consumo y preferencia por la carne bovina & 0,259 & 0,226 & \\
\hline & Considero que la carne de res es una buena fuente de nutrientes & 0,592 & 0,060 & \\
\hline \multirow{5}{*}{$\begin{array}{c}\text { Disposición a comprar carne } \\
\text { bovina con información de calidad } \\
\text { nutricional }\end{array}$} & Importancia de nutrientes & 0,935 & 0,001 & \multirow{5}{*}{-} \\
\hline & Aceptación de la carne bovina & 0,437 & 0,060 & \\
\hline & Estilo de consumo saludable & 0,601 & 0,001 & \\
\hline & Cuando compro carne me fijo en su contenido de grasa & 0,498 & 0,001 & \\
\hline & Me da más confianza comer carne de res, que de pollo o cerdo. & 0,297 & 0,001 & \\
\hline
\end{tabular}


más que por los atributos intrínsecos o extrínsecos de la carne.

\section{Discusión}

Las características socioeconómicas obtenidas en la muestra encuestada son sesgadas con relación a la realidad colombiana, lo cual se puede atribuir al tipo de muestreo empleado (no probabilístico a conveniencia). Esto puede ser comprobado en las diferencias observadas en la proporción hombre/ mujer para la muestra (64/36\%), comparadas con las cifras del DANE (2017), las cuales presentan una proporción de 49,3/50,6\% para hombres y mujeres, respectivamente.

La tasa de respuesta obtenida en la encuesta electrónica se puede considerar baja (18\%). Sin embargo, estos resultados se encuentran dentro del rango publicado por Sheehan (2010), quien argumenta que el uso de encuestas por medio electrónico puede tener un rango de respuestas que oscila entre 6 y $68 \%$, cuya variabilidad está dada principalmente por la edad de los receptores, la longitud de la encuesta, las notificaciones previas, el tiempo de dedicación a sistemas informáticos y la identificación o el prestigio del emisor de la encuesta. Escobar et al. (2015) reportan con el uso de la plataforma Google Form ${ }^{\circledR}$ para una encuesta entre veterinarios vinculados a una asociación de fauna silvestre en Chile, una tasa de respuesta del 36\% (38/107), la cual atribuyen a la ausencia del envío de recordatorios para tener un incremental en la tasa de respuesta, a la edad de los veterinarios que interactuaron con la encuesta y al uso de una lista de correos actualizada, que pudo sesgar la participación de veterinarios de mayor edad, sin datos actualizados en la asociación o sin acceso a correos electrónicos.

Pese a las bajas tasas de respuesta que puede experimentar el uso de encuestas a través de medios electrónicos, esta metodología resulta interesante dada la posibilidad de desarrollar el proceso de investigación a un bajo costo, tal como lo informan Kaplowitz et al. (2004), quienes encontraron que el costo por tasa de respuesta (TR) de encuestas enviadas por correo certificado $(\mathrm{N}=2594, \mathrm{TR}=31,5 \%)$ fue $\$ 10,97$ US, comparado con $\$ 1,32$ US por respuesta cuando la encuesta fue enviada por correo electrónico $(\mathrm{N}=4440, \mathrm{TR}=20,7 \%)$.

Según la muestra encuestada, la calidad intrínseca de la carne estuvo determinada con mayor peso por el color de la carne y la compra del producto en sitios reconocidos. El color de la carne es uno de los atributos considerados como decisivos (primera interacción) por los consumidores a la hora de comprar (Joo et al., 2013), porque es relacionado con una buena conservación del producto (Troy et al., 2010). El color proviene del pigmento hemático mioglobina, el cual es una proteína de $8 \alpha$-helices, un grupo central prostético y una molécula de hierro localizada en la parte hidrofóbica de la proteína. Este atributo intrínseco de la carne puede ser afectado por múltiples factores, entre los que se encuentran el proceso de sacrificio, la interacción del pH en los procesos bioquímicos de transformación de músculo a carne y el almacenamiento del producto (AMSA 2010). Generalmente la mioglobina se encuentra en tres formas: como desoximioglobina, la cual está ligada al hierro y no contiene oxígeno, lo que genera una apariencia de rojo-púrpura en la carne; le sigue la oxymioglobina, la cual se origina por adición de oxígeno a la molécula de mioglobina y produce el color rojo cereza, que los consumidores asocian con carne fresca; y por último está metamioglobina, la cual se genera cuando se oxida la molécula de hierro en la mioglobina y la carne toma un color pardo o rojo oscuro, asociado con baja calidad y poca aceptación del producto (Troy et al., 2010).

Henchion et al. (2017) encontraron en una revisión sobre la percepción de los consumidores respecto a los atributos de calidad para carne bovina que el color se categoriza en importancia como el cuarto atributo de búsqueda para determinar la calidad del producto. En esta investigación, al igual que en otras antes citadas, los consumidores relacionan el color de la carne con la conservación del producto. Sin embargo, el color no es considerado como un atributo definitivo para conceptualizar la calidad de la carne y por consiguiente necesita de otros atributos para soportar su experiencia de compra.

La confianza en el lugar de compra ha sido descrita por varios autores (Grunet et al., 2004) como un atributo extrínseco y de confianza importante para los consumidores al momento de comprar. Este aspecto evalúa la confianza con base en la experiencia de compras previas y en aspectos como la observación de la forma de manipulación de la carne, el empaque de los productos y la cadena de frío, los cuales permiten a los consumidores hacer inferencias sobre la condición sanitaria e inocuidad del producto (Grunet et al., 2004; Troy et al., 2010).

Aunque los resultados encontrados en el presente trabajo no relacionan la grasa de la carne como un 
atributo importante dentro de la calidad intrínseca del producto, varios autores (Grunet et al., 2004; Font-iFurnols et al., 2010; Henchion et al., 2017) citan que la grasa (intramuscular y subcutánea) se considera como un atributo importante de la carne, aunque su efecto generalmente es negativo sobre la percepción de calidad (Joo et al., 2013).

El concepto de grasa de la carne como un atributo de calidad introduce un conflicto de intereses en los consumidores puesto que, desde su concepción como atributo de búsqueda, la grasa se percibe como un factor negativo dado que se asocia con las enfermedades cardiovasculares, entre otras. Sin embargo, desde el punto de vista de la calidad y experiencia de consumo, la grasa se presenta como un atributo importante, dada su participación en el marmóreo, jugosidad, terneza, sabor y olor de la carne (Grunet et al., 2004; Joo et al., 2010; Font-i-Furnols et al., 2010; Henchion et al., 2017). Este conflicto de intereses fue revelado por Morales et al., (2013), quienes señalaron que un mismo corte de carne con alto contenido de marmóreo tenía mayor aceptación de consumo cuando no se informaba su contenido de grasa (aceptación a ciegas), que cuando se informaba (aceptación informada).

Para el caso colombiano, según FEDEGAN (2009), en una encuesta realizada a consumidores de carne bovina, el color de la carne y el sitio de compra se consideran atributos de importancia al momento de realizar la compra y al igual que los resultados presentados en este trabajo, la grasa no es relevante para adquirir el producto.

La preferencia por el consumo de carne bovina, asociada a la variable latente "consumo y preferencia de la carne bovina", ha sido descrita por otros autores como Barrena y Sánchez (2002), quienes informaron que en las comunidades de Navarra y Aragón (España), los consumidores anteponen el consumo de carne bovina al de cerdo y cabra. Así mismo FEDEGAN (2009) informa que en Colombia, el consumo de carne bovina tiene mayor preferencia que el consumo de pollo. Sin embargo, cuando esta preferencia considera la dimensión económica (costo del producto), los consumidores optan por comprar pollo como sustitución directa por el mayor costo de la carne bovina (Santana et al., 2009). En esta investigación, la preferencia por el consumo de carne bovina pudo estar influenciada por el sesgo que presenta la muestra en los ingresos económicos de los encuestados, donde el $37 \%$ declaró recibir más de $\$ 3,6$ millones de pesos al mes, lo cual podría ser un factor que no limite su elección en la compra del producto .

Las variables latentes que indicaron la importancia de la revisión de la información nutricional de los alimentos y la dimensión del estilo de consumo de alimentos saludables, evidenciaron la necesidad que tienen los encuestados de conocer las características de los productos que consumen. Socollan et al. (2006) destacan que la globalización de la información asociada a diferentes enfermedades como el cáncer, la arterioesclerosis y la diabetes genera una presión sobre los consumidores, con un consecuente incremento de su preocupación asociada a la información de los alimentos que incluyen en su dieta. Esta misma presión es ejercida sobre la industria de los alimentos, forzándola a responder a las crecientes demandas del consumidor sobre información de los productos.

Esta situación genera una oportunidad para la industria, y en este caso particular para la industria de la carne, basada en la posibilidad de informar sobre las características nutricionales del producto y con ello potencializar el consumo (Troy et al., 2010). Así quedó evidenciado en la variable latente que indagó sobre la disposición a comprar carne con información de calidad nutricional, en la cual el $68 \%$ de los encuestados respondieron estar dispuestos a pagar más dinero (mediana \$1.000 COP) por tener información nutricional (grasa, proteína y perfil de ácidos grasos).

En el presente trabajo, la disposición a comprar carne con información nutricional estuvo motivada por el comportamiento de revisión de información de los alimentos que consumen los encuestados y por la necesidad de adquirir productos saludables para sí mismos y para su familia, másque por las características intrínsecas de la carne. Este comportamiento sugiere que, más que aceptar la carne por sus características intrínsecas, la disposición de los consumidores a comprar el producto con información de calidad,se daría con base en la credibilidad de la información nutricional y por la expectativa del consumo de la carne y sus efectos en la salud (Henchion et al., 2017).

De manera general, es importante resaltar que en esta investigación los encuestados dimensionan como calidad de carne aspectos como el color y el lugar de compra, y con menor importancia el contenido de grasa, dejando de lado aspectos claves como la terneza, la capacidad de retención de agua y el precio para factores intrínsecos, los cuales estuvieron considerados en el cuestionario, pero no fueron priorizados dentro de las respuestas. Este fenómeno también fue descrito por FEDEGAN 
(2009), que encontró que para la muestra encuestada la calidad de la carne estuvo dada por factores como higiene, refrigeración, fechas de vencimiento, peso e identificación de los productos, distando aún más del concepto científicamente reconocido como calidad de carne (Joo et al., 2013; Henchion et al., 2007). Esta situación está fundamentada en la formación del consumidor y la poca culturización y educación hacia el consumo de carne (Font-i-Furnols et al., 2014), aspecto que demanda un importante esfuerzo de la industria y de la cadena cárnica para investigar sobre la percepción y culturización de los consumidores, con el objeto de generar los conceptos necesarios para que ellos demanden la calidad del producto.

\section{Conclusiones}

El uso de la plataforma Google Form ${ }^{\circledR}$ permitió desarrollar la investigación a bajo costo, haciendo posible enviar, capturar y procesar la información relacionada con el cuestionario desarrollado.
La aplicación de MEE permitió analizar el fenómeno de la percepción de la calidad de la carne, e identificar los factores que más pesan sobre el concepto de calidad de la carne y la disposición a pagar por información nutricional.

El color de la carne y el sitio de compra se constituyeron como los principales factores para conceptuar la calidad de la carne en la muestra encuestada, aspecto que evidencia una necesidad de intervención de la industria en la culturización y educación del consumidor de carne bovina.

La disposición a pagar por información nutricional de la carne estuvo influenciada principalmente por la necesidad del consumo de alimentos saludables, lo cual abre una oportunidad potencial para incrementar el consumo de carne bovina a partir del aporte de información nutricional del producto, aunado al desarrollo de investigaciones que develen el efecto real de la ingesta consumo de carne bovina en la salud humana.

\section{Literatura citada}

American Meat Science Association.

2012. Meat color measurement guidelines (December 2012 Ed.). American Meat Science Association. Champaign, Illinois USA. 133p.

Barrena, R.; Sánchez, M.; Gracia, A.; Gil, J. M.

2002. Confidence in the beef production system as a key factor to mitigate the impact of BSE on beef consumption. $\mathrm{X}$ Congress of the European Association of Agricultural Economists "Exploring Diversity in the European AgriFood System" (August) pp. 1-16.

Cordts, A.; Nitzko, S.; Spiller, A.

2014. Consumer response to negative information on meat consumption in Germany. International Food and Agribusiness Management Review, 17 (Special Issue A), 83-106.

Escobar, L; Yung, V.; Vargas-Rodríguez, R.; Medina-Vogel, G.; Favi, M.

2015. Wildlife veterinarians rabies vaccination in Chile: a survey. Revista Chilena de Infectología : Órgano Oficial de la Sociedad Chilena de Infectología, 32 (3): 289-93.

FEDEGAN - Federación Colombiana de Ganaderos.

2009. Así es nuestro consumidor y así nos percibe. Revista Carta Fedegan, 111: 22-26.

Font-i-Furnols, M.; Guerrero, L.

2014. Consumer preference, behavior and perception about meat and meat products: An overview. Meat Science, 98 (3): 361-371.

Frank, D.; Oytam, Y.; Hughes, J.

2017. Sensory Perceptions and New Consumer Attitudes to Meat. En: Purslow, P. P. (Ed.). New Aspects of Meat Quality: From Genes to Ethics. Elsevier. Hampshire, United Kingdom. pp. 667-698.
Gravetter, F.; Forzano, L.

2015. Research methods for the behavioral sciences. SAGE. Belmont, CA: Wadsworth Cengage Learning. 53p.

Grunert, K.; Bredahl, L.; Brunsø, K.

2004. Consumer perception of meat quality and implications for product development in the meat sector - a review. Meat science, 66 (2): 259-272.

Hair, J. F.; Anderson, R. E., Babin, B. J.; Black, W. C.

2010. Multivariate data analysis: A global perspective. Seventh edition. Pearson. Upper Saddle River, New Jersey, US. 761p.

Henchion, M.; McCarthy, M.; Resconi, V.

2017. Beef quality attributes: A systematic review of consumer perspectives. Meat Science, 128: 1-7.

Joo, S.; Kim, G.; Hwang, Y.; Ryu, Y.

2013. Control of fresh meat quality through manipulation of muscle fiber characteristics. Meat Science, 95 (4): 828-836.

Kaplowitz, M.; Hadlock, T.; Levine, R.

2004. A comparison of web and mail survey response rates. Public Opinion Quarterly, 68 (1): 94-101.

Kline, R.

2011. Principles and practice of structural equation modeling. Ed. Guilford Publications. New York, United States of America. 534p.

Li, X.; Jensen, K.; Clark, C.; Lambert, D.

2016. Consumer willingness to pay for beef grown using climate friendly production practices. Food Policy, 64: 93-106.

Morales, R.; Aguiar, A.; Subiabre, I.; Realini, C.

2013. Beef acceptability and consumer expectations associated with production systems and marbling. Food Quality and Preference, 29 (2): 166-173. 
Navarrete, J.

2000. El muestreo en la investigación cualitativa. Investigaciones sociales, 4 (5): 165-180.

Pereira, P.; Vicente, F.

2017. Meat Nutritive Value and Human Health. En: Purslow, P. P. (Ed.). New Aspects of Meat Quality: From Genes to Ethics. Elsevier. United Kingdom pp. 465-477.

Sales-Campos, H.; Reis de Souza, P.; Crema Peghini, B.; Santana da Silva, J.; Ribeiro Cardoso, C.

2013. An Overview of the Modulatory Effects of Oleic Acid in Health and Disease. Medicinal Chemistry, 13 (2): 201-210.

Santana, A.; Camacho, C.; Estévez, L.; García, G.; Gómez, M.; Gutiérrez, J.; Ballesteros, M.

2009. Agenda prospectiva de investigación y Desarrollo Tecnológico para la Cadena Cárnica Bovina en Colombia. Editorial Giro. Bogotá DC, Colombia. 234p.
Scollan, N.; Hocquette, J.; Nuernberg, K.; Dannenberger, D.; Richardson, I.; Moloney, A.

2006. Innovations in beef production systems that enhance the nutritional and health value of beef lipids and their relationship with meat quality. Meat science, 74 (1): 17-33.

Sheehan, K.

2001. E-mail survey response rates: A review. Journal of Computer-Mediated Communication, 6 (2): 0-0.

Team, R. C.

2016. R: A language and environment for statistical computing. Vienna: R Foundation for Statistical Computing.

Troy, D.; Kerry, J.

2010. Consumer perception and the role of science in the meat industry. Meat Science, 86 (1): 214-226.

USDA

2016. NDB No. 23491 Beef composite, separable lean only, trimmed to $1 / 8$ " fat, choice, cooked. 